\title{
Does extreme mixing improve frequency agreement for $\delta$ Scuti stars?
}

Joyce A. Guzik, Benjamin A. Austin, Paul A. Bradley, Arthur N. Cox

Los Alamos National Laboratory, X-2, MS T085, Los Alamos, NM 87545-2345, USA

\begin{abstract}
Scuti stars that have been observed intensely by the Delta Scuti Network and other campaigns exhibit fewer modes than predicted in the observed frequency range. In addition, some modes are difficult to match by the most likely observable $\ell=0,1$, and 2 modes, especially considering available spectroscopic mode identifications. Here we examine frequency predictions for stellar models for FG Vir and $4 \mathrm{CVn}$ with extensive core mixing, well beyond the plausible limits of such mixing from convective core overshooting. We find that mixing has the potential to substantially improve frequency agreement, and deserves further investigation.
\end{abstract}

\section{Introduction}

Stellar evolution and pulsation modeling with standard physical input that gives excellent agreement for observed solar frequencies fails to reproduce the observed frequencies of $\delta$ Sct stars that have been well observed by the DSN and other campaigns. The model predictions do not fit all of the frequencies simultaneously, especially when taking into account available mode identifications. In addition, far fewer modes are observed than are predicted, if only the likely observable $\ell=0,1$, and 2 modes are considered. We explored whether extending the region of homogeneous core composition beyond that expected from usual core convection, and changing the composition gradient outside the homogeneous core, can improve frequency agreement. The size of the homogeneous core and the composition gradients could be altered by a number of processes, for example, extreme convective overshoot, meridional circulation, rotational shear due to core spin-up and shrinkage at the end of core H-burning, or turbulence generated by shear dissipation of horizontal $g$-mode motions with significant amplitude just outside the convective core. We consider two case studies with extensive observations: FG Vir and $4 \mathrm{CVn}$.

\section{FG Vir}

For FG Vir we use the following observational constraints (see Breger et al. $1999 \mathrm{a}$, and references therein): $\log \mathrm{L} / \mathrm{L}_{\odot}=1.15 \pm 0.05, \mathrm{~T}_{\text {eff }}=7500 \pm 100 \mathrm{~K}$, $\log g=4.0 \pm 0.1$. Twenty nine modes have been detected, and mode identifications $\ell$ and $m$ have been proposed for the highest-amplitude modes (Breger 
et al. 1999a; Viskum et al. 1998; Mantegazza \& Poretti 2002). The rotational velocity is uncertain, but could be as high as $80 \mathrm{~km} \mathrm{~s}^{-1}$ (Mantegazza \& Poretti 2002). We evolved models that match the $\log \mathrm{L}, \log g$, and $\mathrm{T}_{e f f}$, and that have a radial fundamental mode period of $140.7 \mu \mathrm{Hz}$, in accord with the reported mode identification. We then calculated the remaining frequencies for these models, taking into account rotational splitting of the modes into $2 \ell+1$ components, for comparison with the remaining observed modes. For more information on modeling, see Guzik et al. (2000).

We and others have had difficulty matching the $243.7 \mu \mathrm{Hz}$ mode identified as $\ell=2$ (Guzik et al. 2000; Breger et al. 1999a; Templeton et al. 2001). It is also difficult to match the $230.0 \mu \mathrm{Hz}$ mode identified as $\ell=1$, unless a high, possibly differential, rotation rate is adopted. Table 1 lists standard models evolved with no core overshoot for a variety of initial masses, $\mathrm{Y}$ and $\mathrm{Z}$, and predicted $\ell=2$ modes to be compared with the observed modes at 243.7 and $230.0 \mu \mathrm{Hz}$. In this table, "CC" designates convective core. Even though these models start with different initial conditions, since they have the same radial fundamental mode frequency they also have a similar structure, so that the remaining predicted frequencies turn out to be the nearly the same. Only for the $\mathrm{Z}=0.06$ model is the frequency shifted slightly from $\sim 260$ to $255.9 \mu \mathrm{Hz}$, not enough to reach the $243.7 \mu \mathrm{Hz}$ mode or accommodate other observed modes clustered near this frequency at $241.1,245.7$ and $249.4 \mu \mathrm{Hz}$, even including rotational splitting. Mantegazza \& Poretti (2002) conclude that the 243.7 and $230.0 \mu \mathrm{Hz}$ modes are axisymmetric $(m=0)$, so $m=2$ rotational splitting shifts should not be applied.

Table 1. Evolved standard and mixed models for FG Vir.

\begin{tabular}{lcccccc}
\hline Mass $\left(\mathrm{M}_{\odot}\right)$ & $\mathrm{Y}$ & $\mathrm{Z}$ & $\begin{array}{c}\text { Mass }\left(\mathrm{M}_{\odot}\right) \\
\text { C.C. }\end{array}$ & $\begin{array}{c}\mathrm{X} \\
\text { C.C. }\end{array}$ & $\begin{array}{c}\text { Pred. } \ell=2 \\
(\text { Obs. })\end{array} 243.7$ & $(\mu \mathrm{Hz})$ \\
& & & & & \\
& & & & & & \\
Standard Models & & & & & & \\
1.82 & 0.28 & 0.02 & 0.18 & 0.27 & 260.8 & 223.9 \\
1.95 & 0.28 & 0.03 & 0.21 & 0.34 & 261.1 & 224.2 \\
1.70 & 0.38 & 0.03 & 0.18 & 0.22 & 260.0 & 222.2 \\
2.15 & 0.20 & 0.03 & 0.22 & 0.38 & 260.6 & 222.7 \\
2.15 & 0.28 & 0.06 & 0.26 & 0.40 & 255.9 & 220.9 \\
Mixed Models & & & & & & \\
1.82 & 0.38 & 0.03 & 0.33 & 0.23 & 256.4 & 215.0 \\
1.95 & 0.38 & 0.03 & 0.36 & 0.22 & 254.8 & 212.8 \\
2.20 & 0.28 & 0.03 & 0.40 & 0.31 & $252.2,262.9$ & 212.5 \\
2.25 & 0.28 & 0.03 & 0.41 & 0.31 & $248.3,260.8$ & 212.5 \\
2.30 & 0.28 & 0.03 & 0.43 & 0.32 & $243.6,259.6$ & 212.0 \\
\hline \hline
\end{tabular}

We then considered artificial models in which during the evolution we imposed a large opacity multiplier $(\times 2$ to $\times 3$ at the core boundary, ramping to one further out in the envelope) to force an enlarged convective core. The overshooting we induced amounted to $0.8-0.9$ pressure scale heights. Small opacity multipliers and less extreme overshoot do not shift the $\ell=2$ mode prediction 
away from $260 \mu \mathrm{Hz}$ much (Templeton et al. 2001; Breger et al. 1999a). Note also that 2-D hydrodynamic simulations (Deupree 2000) predict only about 0.35 $\mathrm{H}_{p}$ overshooting at the convective core boundary. The lower part of Table 1 summarizes the models with this induced core enlargement. The $2.3-\mathrm{M}_{\odot}$ model with convective core mass $0.43 \mathrm{M}_{\odot}$ does have a predicted $\ell=2$ frequency at $243.6 \mu \mathrm{Hz}$, in addition to an $\ell=2$ mode at $\sim 260 \mu \mathrm{Hz}$. These changes result because of a shifting of the rapid rise in the Brunt-Väisälä frequency just outside the convective core where a steep composition gradient exists that moves the $g$-mode cavity farther out, and changes the placement of $g$-type vs. $p$-type nodes in the eigensolutions. However, this $2.3-\mathrm{M}_{\odot}$ model does not solve all of the mode matching problems. For instance, the model does not match the observed $230 \mu \mathrm{Hz}$ mode by an $\ell=2$ mode, and it is necessary to match this mode instead by a predicted $\ell=1, m=-1$ mode.

If this amount of core convective overshoot is unphysical, what other mixing mechanisms are plausible? FG Vir is in too early an evolutionary state for core contraction to generate shear mixing due to angular momentum transfer. The rotation rate of FG Vir is fairly rapid, but the timescale for classic meridional circulation is only about half the stellar age, so the mixing would be inefficient. Also the composition gradient build-up outside the core could inhibit mixing. $g$ modes appear the most plausible explanation: The $g$-modes with large amplitude eigenfunctions just outside the convective core are predicted to be stable, but they could be excited by stochastic processes. However, it is not clear that shear mixing by a $g$-modes will not also be inhibited by a composition gradient. For FG Vir, mixing is worth further exploration, but while it has potential to improve frequency agreement for some modes, it might make agreement worse for other modes.

\section{3. $4 \mathrm{CVn}$}

For $4 \mathrm{CVn}$ we adopt the following observational constraints (Breger et al. 1999b): $\mathrm{T}_{\text {eff }}=6900 \pm 100 \mathrm{~K}, \mathrm{~L} / \mathrm{L}_{\odot}=31.5(+5 /-4) ; \log g=3.5 \pm 0.1 .4 \mathrm{CVn}$ is fairly rapidly rotating with observed $v \sin i=73 \mathrm{~km} \mathrm{~s}^{-1} .4 \mathrm{CVn}$ has 18 observed frequencies between 54 and $100 \mu \mathrm{Hz}$, while standard models predict several hundred $\ell=0-2$ modes in this range (Guzik et al. 2000). The $80.7 \mu \mathrm{Hz}$ mode is identified as a radial mode. Four identified $\ell=1$ modes are evenly spaced with an average spacing of $13.5 \mu \mathrm{Hz}$.

Standard $4 \mathrm{CVn}$ models have exhausted core $\mathrm{H}$ and are shell $\mathrm{H}$-burning by the time they reach $T_{\text {eff }}=6900 \mathrm{~K}$. This structure with no convective core allows a central $g$-mode cavity, and greatly increases the number of closelyspaced predicted modes.

Dziembowski (1997; see also Breger \& Pamyatnykh 2002) proposed a partial solution to the mode selection and spacing problems by hypothesizing that the star selectively exhibits modes that are at a kinetic energy minimum (mode trapping). This explanation shows promise, but it is not clear that the modes with lowest kinetic energy should be the only modes visible (this does not hold true, for example, for white dwarf stars). In addition, some of the lower-frequency observed "trapped" modes have higher kinetic energy than do some higherhigher-frequency unobserved modes. 
We are able to evolve a self-consistent model of $4 \mathrm{CVn}$ that has not completely exhausted core $\mathrm{H}$, and therefore has a convective core, by inducing mixing using the same $\kappa$ multiplier during the evolution as in the $2.3-\mathrm{M}_{\odot}$ mixed FG Vir model. This $2.5-\mathrm{M}_{\odot}$ mixed model barely reaches the location of $4 \mathrm{CVn}$ during its evolution redward in the HR diagram before turning around to the blue. It has a convective core mass $0.37 \mathrm{M}_{\odot}$, convective core hydrogen abundance $\mathrm{X}=0.0884$, and an age of $1.6 \mathrm{Gyr}$, compared to $0.88 \mathrm{Gyr}$ for a normal model. For the mixed model, the number of predicted $\ell=0-2$ modes in the observed frequency range is reduced to 30 , compared to over 150 for the normal model. The frequency spacing between $\ell=1$ modes of the mixed model is increased to $\sim 15 \mu \mathrm{Hz}$, compared to $\sim 3 \mu \mathrm{Hz}$ or less for the normal model.

In the case of $4 \mathrm{CVn}, g$-modes could also cause this kind of mixing. Also, during core collapse and $\mathrm{H}$ exhaustion, perhaps rotational shear could mix hydrogen back into the core and make it convective again. We note that $82 \%$ of core angular momentum must be lost to maintain a constant rotation rate during the "blue hook".

\section{Conclusions}

Extreme mixing has the potential to help improve frequency agreement for $\delta$ Sct stars. Mixing is probably occurring to some extent, given the rapid, probably differential, rotation in these stars and possibility of stochastic excitation of $g$ modes. No satisfactory models have been found yet for the most well-observed $\delta$ Sct stars, taking into account the observational constraints and mode identifications. It appears that we do not understand core evolution for intermediatemass stars well enough, and cannot learn about the physics we are getting wrong from comparisons to the sun. This is a very worthwhile puzzle to solve, and we should not be content with superficial agreements for some modes.

\section{References}

Breger, M., Pamyatnykh, A.A., Pikall, H., Garrido, R. 1999a, A\&A, 341, 151

Breger, M., et al. 1999b, A\&A, 349, 225

Breger, M., Pamyatnykh, A.A. 2002, in ASP Conf. Ser., Vol. 259, Radial and Nonradial Pulsations as Probes of Stellar Physics, eds C. Aerts, T.R. Bedding \& J. Christensen-Dalsgaard (San Francisco: ASP), 388

Deupree, R.G. 2000, ApJ, 543, 395

Dziembowski, W.A. 1997, in Sounding Solar and Stellar Interiors, eds J. Provost \& F.-X. Schmider (Netherlands: IAU), 317

Guzik, J.A., Bradley, P.A., Templeton, M.R. 2000, in ASP Conf. Ser., Vol. 210, Delta Scuti and Related Stars, eds M. Breger \& M.H. Montgomery (San Francisco: ASP), 247

Mantegazza, L., Poretti, E. 2002, A\&A, 396, 911

Templeton, M., Basu, S., Demarque, P. 2001, ApJ, 563, 999

Viskum, M., et al. 1998, A\&A, 335, 549 